\title{
TECHNIQUES FOR PUMP-PROBE SYNCHRONISATION OF FSEC RADIATION PULSES
}

\author{
H. Schlarb, DESY, D-22607 Hamburg, Germany*
}

\section{Abstract}

The production of ultra-short photon pulses for UV, VUV or X-ray Free-Electron Lasers demands new techniques to measure and control the arrival time of the FELpulses. For pump-probe experiments using optical-lasers, the desired synchronization between the pump and the probe pulses is on the order of tens of femtoseconds. This corresponds to the typical duration of the FEL pulses. Since accelerators are large scale facilities with a length of several hundred meters or even kilometers, the problem of synchronization has to be attacked twofold. First, the $\mathrm{RF}$ acceleration sections upstream of the magnetic bunch compressors need to be stabilized in amplitude and phase to high precision. Second, the remaining electron beam timing-jitter needs to be determined with femtosecond accuracy for off-line analysis. In this paper, several techniques of measuring the FEL beam arrival-time are presented.

\section{INTRODUCTION}

The study of the evolution of ultrafast systems in the areas of atomic physics, chemistry, biology or condensed matter is typically carried out with pump-probe configurations. The time-dependent phenomena is initiated by a high-power pump pulse, e.g. with an ultrafast optical laser or a laser driven source, and then probed after a defined time delay. By repeating the experiment for different delays, the system changes can be recorded and the underlying dynamics understood.

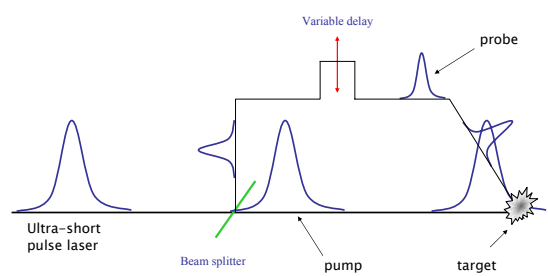

Figure 1: Standard layout of a pump probe experiment.

In the standard setup, shown in Fig. 1, the pump and the probe beam have a common source, so that precise time delays can be produced with optical path-length differences. The time-resolution can be as short as a fraction of a femtosecond and is limited only by the overlap of the pump and the probe pulses $[1,2]$.

While many research activities use femtosecond, highpower optical-lasers for the excitation of the processes,

\footnotetext{
*holger.schlarb@desy.de
}

the spectroscopy resolution provided by the long, optical wavelength cannot determine atomic or molecular displacements.

To provide intense, high energy photons with a wavelength in the VUV, soft, or hard X-ray regime, electrons in the $\mathrm{GeV}$ range are passed through long magnetic undulators. The duration of the photon-pulse at the exit of the undulator is determined by the length of the emitting electron bunch. Ultrashort photon pulses, significantly shorter then the electron pulse, can be created by manipulating the electron bunch spatially, e.g. by impressing a time-angle correlation, using a crab cavity [3], or by introducing an energy variation in a femto-slice and passing it through an undulator placed in a dispersive beam-line [4].

The peak brilliance of the photon-pulse is increased by ten orders of magnitude with Free Electron Lasers. To initiate the lasing process and to achieve saturation, the electron beam has to meet stringent requirements (normalized emittances of $1 \mu \mathrm{m}$ and peak currents of several $\mathrm{kA}$ ). When only a small longitudinal fraction of the electron bunch serves for lasing, photon pulse duration in the femtosecond $[5,6,7]$ or even attosecond regime $[8,9]$ can be achieved.

The layout of an accelerator driving a Free Electron Laser is sketched in Fig 2. The first components is an $\mathrm{RF}$ photo-injector to produce the high brightness electron beam by impinging a short pulse laser $(\approx 10 \mathrm{ps}$ FWHM $)$ onto a photo-cathode in an RF cavity. Due to strong spacecharge forces only moderate peak currents can be produced to preserve the small emittance in a photo-injector. To further increase the peak current and to reduce the electron bunch length, the booster accelerator is operated off-crest. The induced energy chirp along the bunch provides longitudinal compression by an energy dependent path-length in a magnetic chicane. The main linac raises the energy of the electrons to generate photons with the desired wavelength. The length of the accelerator can range from hundreds of meters to several kilometers. Thus, for pump-probe experiments, a second distinct laser-system is used, which is located in the X-ray experimental hall close to the target being studied.

\section{SOURCE FOR TIMING JITTER}

For pump-probe experiments any timing-jitter that effects the relative delay between the laser (pump) and the Xray pulse (probe) at the location of the target has to be taken into account. To synchronize the various sub-systems in a large-scale accelerator, the standard technique is to distribute RF signals in temperature stabilized co-axial cables. A low phase noise master oscillator operated in the $100 \mathrm{MHz}$ range serves as a clean reference. 


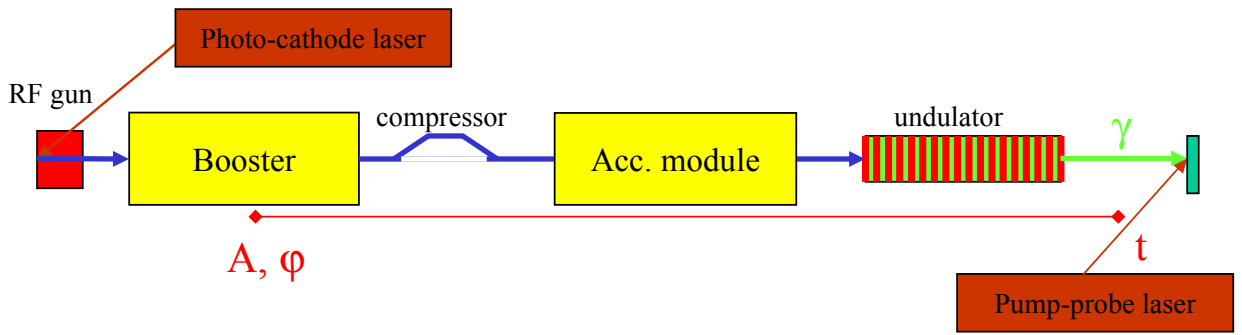

Figure 2: Scheme of an linac driven light source for ultra-short photon pulse production.

The sub-picosecond beam synchronization is degraded by noise, thermal drifts, and electro-magnetic interference in the RF devices. Sub-systems that influencing the arrival time and the stability of the electron beam are the

- photo-cathode laser,

- phase and amplitude of RF gun,

- phase and amplitude of booster,

- magnets in chicane,

- pump-probe laser system,

- laser and photon transport beam line.

The most critical sources for timing-jitter are the RF amplitude and phase jitter of the booster section upstream of the bunch compressor. The longitudinal dispersion $R_{56}$ introduced in the chicane turns energy jitter into timing jitter downstream of the compressor. The timing-jitter in a somewhat simplified model can be written as

$\frac{\Sigma_{t, f}^{2}}{c_{0}^{2}}=\left[R_{56}\left(\frac{\sigma_{A}}{A}\right)^{2}+\left(\frac{C-1}{C}\right)^{2}\left(k_{r f} \sigma_{\phi}\right)^{2}+\left(\frac{1}{C}\right)^{2} \frac{\Sigma_{t, i}^{2}}{c_{0}^{2}}\right]$

where $A$ and $\phi$ are the acceleration amplitude and phase of the booster. $\Sigma_{t, i}$ denotes the timing-jitter coming into the booster and $C=\sigma_{z, i} / \sigma_{z, f}$ is the compression factor given by the ratio of the initial and the final bunch length.

In Eq. 1 it is assumed that the incoming beam energy and energy chirp are much smaller than the energy gain and energy chirp introduced by the booster. Collective effects such as wakefields or coherent synchrotron radiation in the dipole magnets are not taken into account ${ }^{1}$. The residual energy-spread of the beam exiting a photo-injector can be neglected. Higher-order effects due to the non-linearity of the RF curvature or dispersion of the chicane increase the complexity of the equation but do not change the fundamental scaling.

For FELs, compressions between 5 and 20 are used in a single bunch-compression stage. For such large compressions, the timing-jitter from the injector is effectively reduced by a factor of $1 / C$. The most critical parameter is

\footnotetext{
${ }^{1}$ Small wakefields or CSR effects change mainly the operating point of the booster, but do not change significantly the considerations on the timing jitter budget. Large wakefields e.g. used to compress the beam cause that the incoming timing jitter is not compressed anymore.
}

then the RF phase-jitter, which is difficult to control and usually determines the arrival time of the beam at the end of the linac. The RF amplitude jitter can also critically contribute to the timing, but its sensitivity can be reduced by using smaller momentum compaction factors at the cost of more acceleration units to increase the energy chirp ${ }^{2}$.

RF amplitude and phase-jitter of the gun or a timing jitter of the injector laser may degrades beam quality and the beam stability, but does not directly introduce timing-jitter at the experiment.

The Eq. 1 also holds if a second bunch-compressor is implemented (with the same assumptions), but now the jitter introduced by the first compressor is reduced and the RF jitter of the second booster section dominates the electron beam arrival-time.

Therefore, the control and stabilization of RF phase from the last acceleration section energy chirping the electron beam for the final compression defines the arrival time. If an arrival timing stability is required to the $10 \mathrm{fs}$ level, then the RF phase has to be stabilized to $10 \mathrm{fs}$.

Currently, beam timing-jitter of $1 \mathrm{ps}$ to $100 \mathrm{fs}$ is achieved, while slow timing drifts are often much larger and have to be removed with slow feedback loops.

\section{DETECTION OF PHOTON ARRIVAL TIME}

For pump-probe experiments, knowing the relative arrival time between the pump (laser) and the probe beam (e.g. X-ray) at the sample is crucial.

Since the pulses originate from different sources timingjitter of the adjustable delay between pump and probe pulse will be unavoidable. The lack of control can be overcome if one can measure the delay shot-by-shot and with high precision. The experimental data can then be post-ordered according to the measured delay.

Knowledge of the RF phase (and amplitude) in the booster and the timing of the optical laser is, in general, sufficient to determine the time delay. The more direct way,

\footnotetext{
${ }^{2}$ Too large beam energy spreads cause in addition difficulties for the precise energy measurement required for beam based RF feedback loops.
} 
however, is to measure the photon-pulse arrival-time with respect to the pump laser.

\section{Surface Interaction}

Electronic devices such as oscilloscopes or photodetectors are far to slow to determine the arrival times with femtosecond resolution. A standard technique to overcome the electronic resolution limits is to map the temporal information into spatial information which is read out by a slow device.
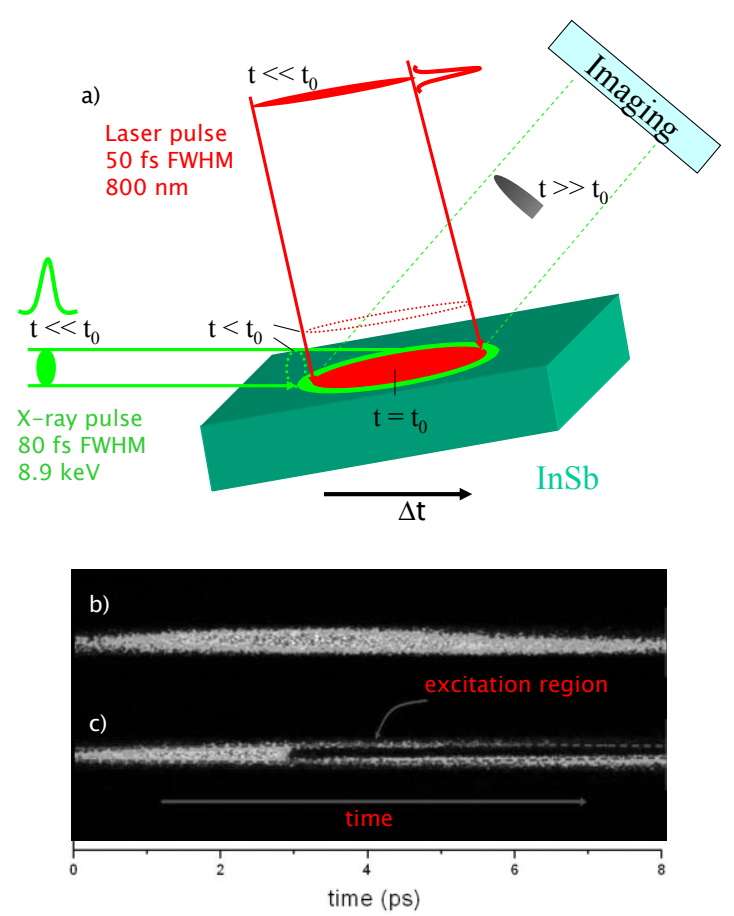

Figure 3: Cross beam geometry.

Figure 3(a) shows the cross-beam geometry used at the Sub-Picosecond Pulse Source (SPPS) at SLAC to detect the arrival time of a $80 \mathrm{fs} \mathrm{X}$-ray pulse [10]. The $8.9 \mathrm{keV}$ photon beam incident at grazing angle onto an asymmetrically-cut indium antimonide (InSb) crystal. Due to the shallow angle, the X-ray pulse impinges the crystal surface at different times. With an optical pump, incident at an angle with respect to the $\mathrm{x}$-ray probe-pulse, the temporal information is turned into spatial information through the difference in propagation times across the sample surface.

The ultra-fast encoding is achieved by a non-thermal melting process at the surface of the InSb substrate, initiated by the intense Ti:Sa femtosecond laser. Within the laser excitation depth of about $50 \mathrm{~nm}$, corresponding to the penetration depth of the X-ray beam, a dense electron-hole plasma is created. The plasma changes the interatomic potential and leads to a rapid disordering of the crystalline lattice. At the surface with disordered lattice one observes are reduced diffraction of the X-ray beam into the camera. Figure 3(b) shows the bright spot of the X-ray image if no laser impinges and in Fig. 3(c) the shadows due to the nonthermal melting process.

Transient times from maximum to minimum reflection as short at $200 \mathrm{fs}$ have been observed. The delays could be determined with precision of $50 \mathrm{fs}$. The intense laser pulse, however, destroys the crystal structure of the InSb semiconductor and the method cannot be used for high photon repetition rates.

\section{Cross-Correlation}

A cross-correlation technique has been proposed to monitor the intensity profile and the arrival time of the VUVFEL pulses at DESY [12]. The FEL beam, with wavelengths between $6 \mathrm{~nm}$ and $100 \mathrm{~nm}$, crosses an atomic gascell. In the gas, photoelectrons are produced with a kinetic energy determined by difference of the FEL radiation energy and the binding energy of the electrons to the atoms. The spectral width of the photoelectrons is equal the FEL bandwidth and amounts to about $1 \%$.

The photoelectrons produced in the gas are mapped transversely onto a CCD-line camera. A dispersive electron lens-system, with an energy acceptance smaller or comparable to the bandwidth of the photoelectrons is used. To extract the time profile and the arrival timing of the VUVradiation, an ultrashort laser pulse is injected perpendicular to the FEL beam (cross-geometry configuration). The intense laser field causes a photon-line broadening in the gas volume where the laser and the VUV photons overlap. Because of the limited energy acceptance of the dispersive lens-system, now only a small fraction of the born photoelectrons will be imaged onto the camera.

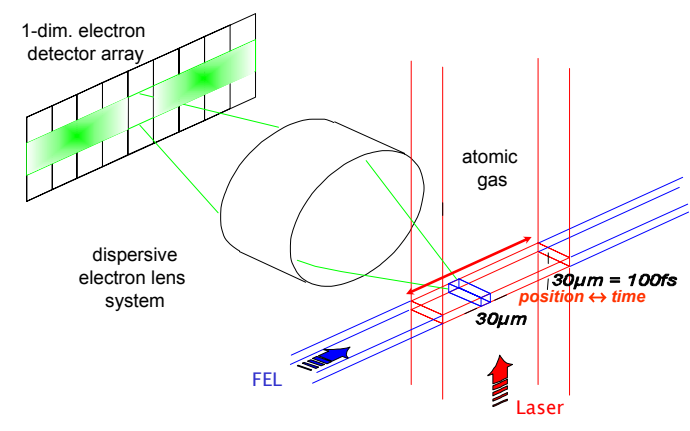

Figure 4: Cross-correlation setup.

The cross-correlation technique allows to detect shot-byshot the FEL pulse profile and the FEL delay with respect to the laser at high repetition rates. Due to small attenuation of FEL beam in the gas-cell, the system can be used as a permanent monitor while pump probe experiments are carried out.

\section{DETECTION OF THE ELECTRON ARRIVAL TIME}

A different approach for detecting the delay time for pump-probe experiments is to determine the electron bunch 
arrival at the undulator. For FELs, one has to assume that the electron beam properties within the bunch are preserved, such that longitudinal variation of the part of the electron bunch contributing to lasing process can be neglected. The time of the FEL radiation exiting the undulator is given for example by the center of mass of the electron bunch, and only the timing-jitter due to the photon transport line to the experiment can accumulate.

The most frequently used method to determine the arrival time of the electron beam is to detect the phase of a beam induced RF signals, for instance extracted from a resonant cavity, which is compared to the RF phase from the linac reference.

To avoid additional timing-jitter due to the reference line, the arrival time of the electron bunches can be directly measured using the optical laser in an electro-optical setup.

\section{Electro-Optic Sampling Technique}

An electro-optic (EO) experiment permits to measure the time structure of electron bunches with high resolution by detecting the transient electric field of the relativistic electrons entering a nonlinear electro-optic crystal. The induced polarization anisotropy in the EO-crystal, e.g. ZnTe, is determined through ellipsometry using synchronized femtosecond laser pulses. Sampling of the copropagating electric field is performed by scanning the delay time of the laser relative to the electron bunch. Figure 5 shows the main components of an EO-experiment[13].

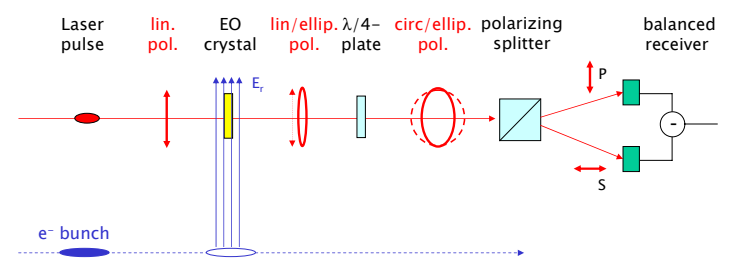

Figure 5: Detection scheme for electro-optical measurements.

\section{Single Shot EO-Configuration}

The sampling scheme is a multi-shot method and does not provide the arrival time information reliably. EOtechniques allowing single-shot electron-beam profiling and timing measurement are sketched in Fig. 6(a)-(c).

Frequency domain: The ultrashort laser-pulse is stretched using a linear dispersive medium or a prism compressor. The pulse duration is chosen to guarantee always an overlap of the laser with the electron bunch. The stretching process introduces a linear, time-spectral correlation to the laser pulse. The time profile of the electron bunch is encoded onto the intensity envelope of the chirped optical pulse and subsequently decoded through a single-shot
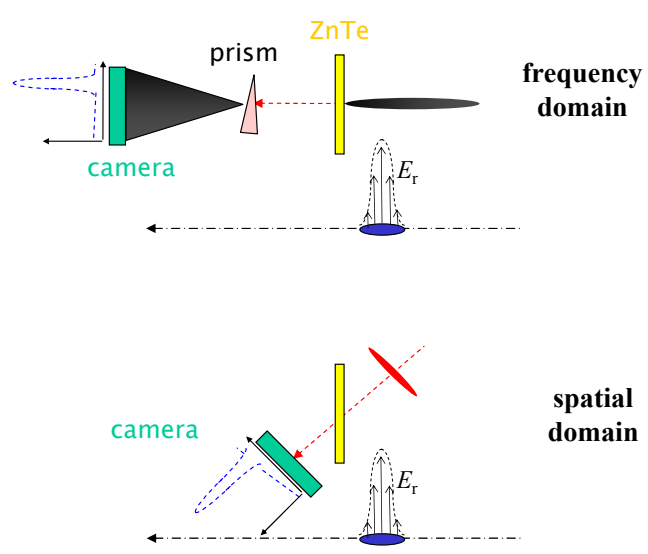

spatial

domain

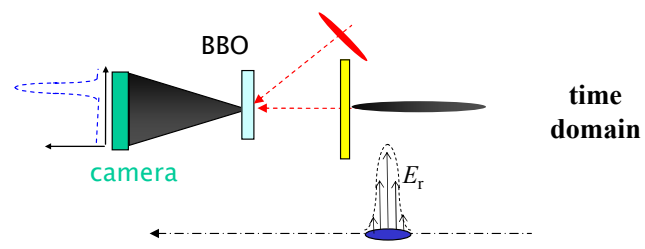

Figure 6: Electro-optic techniques for single shot measurements.

measurement of the pulse spectrum [14]. Due to the limited bandwidth of the laser, the spectral decoding process has an intrinsic time-resolution limitation of about $0.5 \mathrm{ps}$ (FWHM) [15].

Spatial domain: The femtosecond laser-beam incident on the crystal at an angle to the electron-beam axis. The top edge of the pulse intersects the EO-crystal before the bottom edge. This encodes the time to the spatial coordinate across the diameter of the laser spot. By replacing the balanced detector with a fast CCD (line-) camera, the electron beam profile and arrival time can be measured in a single shot. The method is intrinsically limited by either the resolution of the optical system imaging the crystal onto the CCD (about $30 \mathrm{fs}$ ) or by the laser-pulse duration. The fastest EO signal observed sofar had a pulse duration of $300 \mathrm{fs}$ (FWHM) [16].

Time domain: The high-power short pulse laser is split into two beams. One beam serves as a short-pulse reference beam in a single-shot cross-correlator. The other beam is stretched before passing the EO-crystal collinear to the electron beam. The electric field of the electron bunch is encoded in the polarization of the stretched laser, then converted, with a quarter-wave plate and polarizer to an intensity modulation. In the cross-correlator, both laser beams are overlapped spatially in a $\beta$-barium borate (BBO) second-harmonic-generation crystal. Non-collinear incidence of the beams allows for detection of the temporal intensity modulation in a single shot with a CCD camera [17]. The intrinsic resolution limitation of the method is 
given by duration of the reference laser pulse (typically $30 \mathrm{fs}$ FWHM). The single shot cross-correlator requires pulse energies of several hundred $\mu \mathrm{J}$. This limits the laser repetition rate to about $10 \mathrm{kHz}$.

\section{Resolution Limitation Due to EO-Crystal}

Detailed numerical studies on the limitations of the electro-optic measurements for zinc telluride ( $\mathrm{ZnTe})$ and gallium phosphide $(\mathrm{GaP})$ can be found in [18]. The most severe limitation on the time resolution is given by the transverse optical lattice oscillation in the EO-crystal. The frequency dependence of the refraction index, and the electro-optic coefficient, as well as, phase slippage between the laser group and $\mathrm{THz}$ phase velocity of the electric field broaden the pulse duration detected by the setup. It has been found, that the shortest electron bunch length which can be resolved with moderate distortion amounts to about $200 \mathrm{fs}$ (FWHM) for ZnTe and $100 \mathrm{fs}$ for GaP.

All electro-optic techniques presented here are noninterceptive and are well suited for permanent monitoring of the accelerator performance.

At SPPS, the spatial domain EO technique has been successfully used to determine the delay time between the Xray probe and the pump laser pulse with a precision of $30 \mathrm{fs}$ (FWHM) by detecting the electron bunch arrival time [16]. Good agreement was found by correlating the data with the photon arrival time monitor using the non-thermal melting technique.

\section{FUTURE SYNCHRONIZATION SYSTEM}

In order to reduce the timing jitter directly at the source, synchronization systems based on the distribution of optical signals have been proposed $[19,20]$. The layout of a system distributing laser pulses is sketched in Fig. 7.

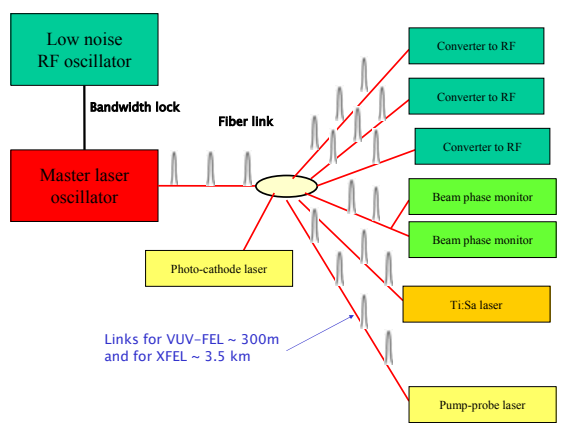

Figure 7: Next generation synchronization system.

A passive mode-locked, ultra-stable Erbium-doped fiber laser with a repetition rate $f_{0}$ (typically $30-60 \mathrm{MHz}$ ) is locked to an ultra-low noise RF master oscillator. Laser pulses of about $0.5 \mathrm{ps}$ duration with a central wavelength of $1550 \mathrm{~nm}$ are distributed in optical path-length stabilized fiber links. At the end of the link, the laser pulses are converted to an RF frequency (any multiple $f_{0}$ ), used to di- rectly synchronize laser systems (based on sum harmonic generation), and to monitor the electron bunch arrival time. At the VUV-FEL it is planned to develop and test a prototype version with the capability to synchronize the various points in the machine with a stability of better than $10 \mathrm{fs}$ (rms)[19].

\section{SUMMARY}

The precise knowledge of the delay between the pump and the probe beam to study fast transient processes is essential for the success of the experiments. Timing jitter of several tens femtoseconds between the optical lasers and the electron beam produced in an accelerator driving a FEL may is unavoidable. To overcome the lack of control, various detections schemes have been presented to directly access, shot-by-shot, the timing-jitter which allows the postordering the experimental data.

\section{REFERENCES}

[1] M. Hentschel et al., "Attosecond metrology," Nature 414, 509-513 (2001).

[2] E. Goulielmakis et al., "Direct Measurement of Light Waves," Science, Vol. 305, 1267-1269 (2004).

[3] J.N. Corlett et al., "A Recirculating Linac-Based Facility for Ultrafast X-ray Science,” PAC'03, May 2003, Portland.

[4] R.W. Schoenlein et al., "Generation of Femtosecond Pulses of Synchrotron Radiation," Science 287, 2237 (2005).

[5] V. Ayvazyan et al., Phys. Rev. Lett. 85, 38253829 (2000).

[6] V. Ayvazyan et al., Phys. Rev. Lett. 88, 104802 (2002).

[7] B. Faatz, "First Results from the VUV FEL at DESY," PAC'05, May 2005, Tennessee.

[8] P. Emma et al., Phys. Rev. Lett. 92, 074801 (2004).

[9] A. Zholents, "Methods of Attosecond X-Ray Pulse Generation," PAC'05, May 2005, Tennessee.

[10] A. M. Lindberg et al., "Atomic-scale visualization of inertial dynamics," to be published in Science (2005).

[11] J. Hastings et al., "Sub-Picosecond Pulse Source: Recent Results," PAC'05, May 2005, Tennessee.

[12] M. Drescher, DESY Proposal II-02-042 FEL.

[13] G.M.H. Knippels et al., Opt. Lett., Vol. 23, 1754 (1998).

[14] I. Wilke et al., Phys. Ref. Lett. 88, 124801 (2002).

[15] S.P. Jamison et al., Opt. Lett., Vol. 28, 1710 (2003).

[16] A. Cavalieri et al., Phys. Ref. Lett. 94, 114801.

[17] G. Berden et al., Phys. Ref. Lett. 93, 114802 (2004).

[18] S. Casalbuoni et al., "Numerical Studies on the ElectroOptic Sampling of Relativistic Electron Bunches," TESLA Report 2005-01.

[19] F. Kaertner et al., "Progress in Large Scale Femtosecond Timing Distribution and RF-Synchronization," PAC'05, May 2005, Tennessee.

[20] R. Wilcox et al., "Optical Synchronization Systems for Femtosecond X-Ray Sources,” PAC'05, May 2005, Tennessee. 